MATEC Web of Conferences 22,02003 (2015)

DOI: $10.1051 /$ matec conf/ 20152202003

(C) Owned by the authors, published by EDP Sciences, 2015

\title{
Research on the Collinear Equation Model of Visual Positioning Based on Visible Light Communication
}

\author{
Yuqi Wang \\ Academy of OPTO-Electronics, Chinese Academy of Sciences, Beijing, China \\ University of Chinese Academy of Sciences, Beijing, China \\ Yingkui Gong* \\ Academy of OPTO-Electronics, Chinese Academy of Sciences, Beijing, China \\ Zhengfa Shi \\ Academy of OPTO-Electronics, Chinese Academy of Sciences, Beijing, China \\ University of Chinese Academy of Sciences, Beijing, China
}

\begin{abstract}
A positioning method based on visible light communication is proposed, which receiving visible light information by low-resolution photodiode array and receiving visual information by the front camera of mobile phone. The terminal position is determined by matching spot information provided by photodiode array with visual information and position information provided by visible light communication. A collinear equation model is derived which based on mobile phone front camera. A hardware-in-loop simulation has been conducted to verify the collinear equation. The three-dimensional positioning error is on the level of decimeter. Moreover, the main factors which affect the positioning accuracy are analyzed in order to further improve the positioning accuracy.
\end{abstract}

Keywords: collinear equation; visual positioning; simulation and analysis; visible light communication

\section{INTRODUCTION}

With the continuous development of visible light communication technology, its high-speed, wide-bandwidth and no electromagnetic radiation characteristics have brought new opportunities for indoor navigation and positioning. Visible light communication can provide communication services in the electromagnetic constrained scenarios such as airplane, hospitals and mines, moreover, its high-precision navigation and positioning services bring a broad development. Currently, indoor positioning technology based on visible light communication can be divided into non-visual and visual positioning. Non-visual technology includes LED identification positioning ${ }^{[1]}$ (LED-ID), time difference of arrival positioning ${ }^{[2]}$ (TDOA), angle of arrival positioning ${ }^{[3]}$ (AOA) and received signal strength positioning ${ }^{[4]}$ (RSS). Two methods of visual positioning technology are proposed currently. One is the double image sensor positioning scheme which using more than 4 different colors of light to distinguish the optical signals ${ }^{[5]}$, the other positioning scheme is using the collinear relationship between multiple lamps with same sensor to get the terminal position ${ }^{[6]}$.

In this paper, a double-visual-projection positioning method is proposed, which uses a photodiode (PD) array and the mobile terminal front camera as image sensor to image the LED lights. Due to the imaging principle of mobile terminal front camera and the space coordinates relationship between mobile terminals and LEDs which are different from traditional photogrammetry methods, mathematical model is not the same. Therefore, this paper put forward a collinear equation model suiting for indoor positioning with mobile terminal front camera, and a hardware-in-loop simulation has been conducted to verify the collinear equation.

\section{METHOD AND MODEL OF POSITIONING}

\subsection{Method of positioning}

Indoor positioning system based on visible light communication in this paper is designed. LED lights installed by a certain arrangement on the ceiling, which are used power line carrier to connect with the control center in the background, and the control center sends positioning information of each lamp at a certain time interval. The mobile terminal with external PD array receives the LED light spots and the visible light communication information. And at the same time, the mobile terminal takes LED image through the mobile front camera. The visible light communication using LED can provide spot mapping and LED location information, and the mobile phone camera can provide high-resolution LED image. Due to

*Corresponding author: ykgong@aoe.ac.cn

This is an Open Access article distributed under the terms of the Creative Commons Attribution License 4.0, which permits unrestricted use, distribution, and reproduction in any medium, provided the original work is properly cited. 


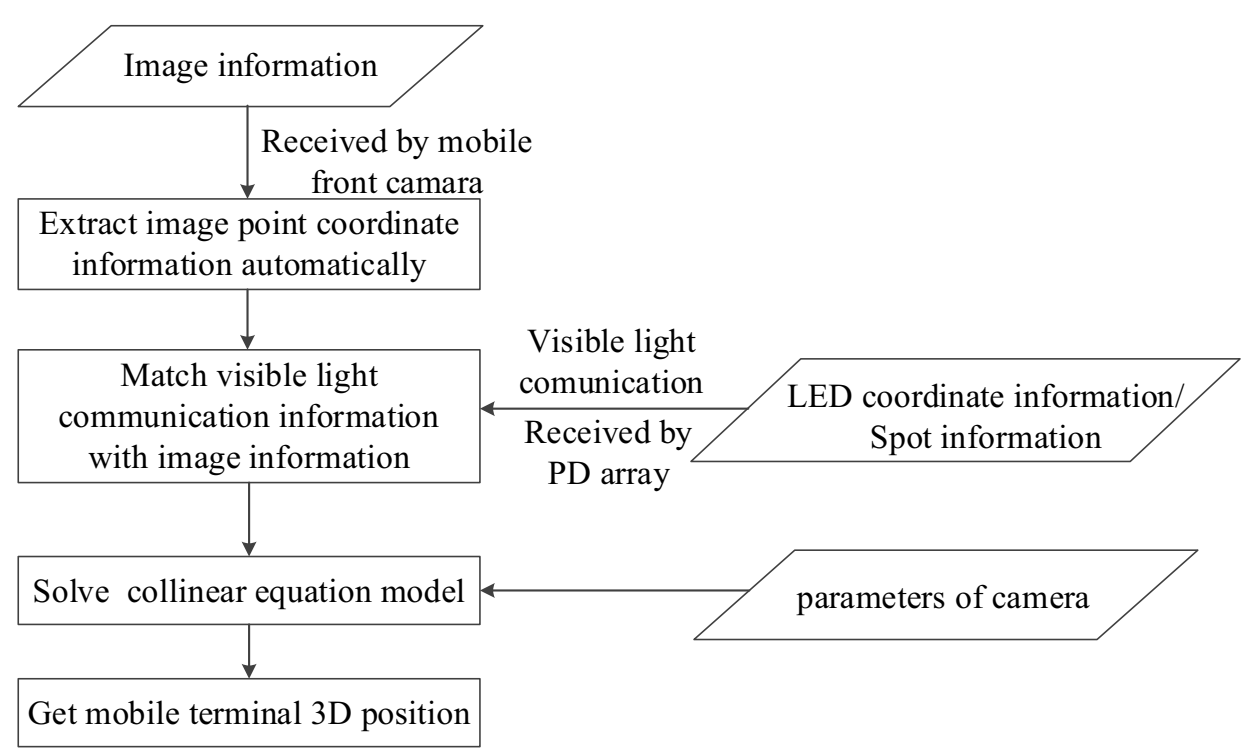

Figure 1. Process of positioning

the low-resolution spot information is insufficient for positioning, the spot position information needs to match the imaging information taken by mobile terminal in order to obtain mobile terminal's positioning. Visual-based positioning method based on visible light communication adopted multiple LED lamps for real-time decoding, and it needn't the time synchronization. The process of positioning is shown in Figure 1.

The coordinates of LED lights on the image are extracted after obtaining the visual information of LED lights. Meanwhile, we obtain the three-dimensional coordinate information of LED lights by using PD array and the spot in PD array through visible light communication. Match the three-dimensional coordinate information with visual information, and establish error equation based on collinear equation model. Thus, three-dimensional coordinates of the mobile terminals can be calculated by inputting related parameters of the camera

\subsection{Collinear equation model based on mobile front camera}

The geometric model of light should be analyzed and derived if the collinear equation model is used for the positioning scene in Chapter 2.1. First, the location relationship among LED light, image point and photographic center is analyzed. Then, the relationship between the vision information and the optical imaging is obtained by analyzing the mobile front camera.

LED light, image point and photographic center are in a direct line according to the characteristic of image projection in positioning. The $\mathrm{Z}$ coordinate of terminal is always less than that of LED lights, so the photo auxiliary coordinate system and the photo coordinate system are established for convenience. The center of the photo auxiliary coordinate system is at the photo- graphic center and the axes are parallel to the coordinate axes in the LED three-dimensional coordinate system while the $\mathrm{z}$ axis is in the opposite direction. The center of the photo coordinate system is the center of image which is always the projection of photographic center in image and the coordinate axes is parallel to the coordinate axes in the photo auxiliary coordinate system.

The imaging relationship is derived as follows for the model:

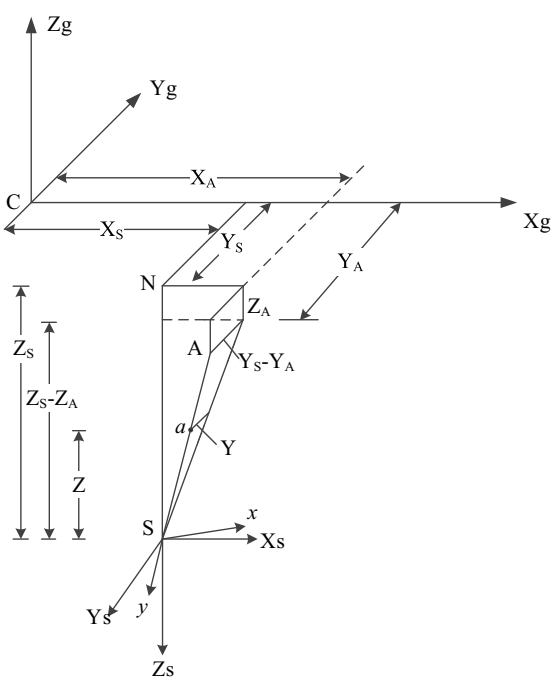

Figure 2. Collinear equation model based on mobile phone

In Figure 2, A is LED light, $a$ is the image point of LED light, and $\mathrm{S}$ is the photographic center. 
$C$ - $X g Y g Z g$ is the coordinate system of LED, $S-X_{s} Y_{s} Z s$ is the image space auxiliary coordinate system of camera and $S$-xy is image coordinate system. There are 3 angles between the mobile terminal and the axis of image space auxiliary coordinate system. Assume that $\varphi, \omega, \kappa$ are respectively the directional dip, the lateral rotation angle and the image dip of terminal respectively in y axis. According to the geometric relations, if the mobile phone terminal rotation is parallel to the coordinate axes, then the rotation matrix is $\mathrm{R}=\left(a_{1}, a_{2}, a_{3}, b_{1}, b_{2}, b_{3}, c_{1}, c_{2}, c_{3}\right)$, where:

$$
\left\{\begin{array}{l}
a_{1}=\cos \varphi \cos \kappa-\sin \varphi \sin \omega \sin \kappa \\
a_{2}=-\cos \varphi \sin \kappa-\sin \varphi \sin \omega \cos \kappa \\
a_{3}=-\sin \varphi \cos \omega \\
b_{1}=\cos \omega \sin \kappa \\
b_{2}=\cos \omega \cos \kappa \\
b_{3}=-\sin \omega \\
c_{1}=\sin \varphi \cos \kappa+\cos \varphi \sin \omega \sin \kappa \\
c_{2}=-\sin \varphi \sin \kappa+\cos \varphi \sin \omega \cos \kappa \\
c_{3}=\cos \varphi \cos \omega
\end{array}\right.
$$

In order to derive conveniently, the scaling factor is introduced. Assume that:

$$
\left[\begin{array}{c}
X \\
Y \\
Z
\end{array}\right]=\frac{1}{\lambda}\left[\begin{array}{c}
X_{A}-X_{S} \\
-Y_{A}+Y_{S} \\
-Z_{A}+Z_{S}
\end{array}\right]
$$

Then the relationship of image point between photo coordinate system and LED coordinate space is shown as follows:

$$
\left[\begin{array}{l}
x \\
y \\
-f
\end{array}\right]=R^{T}\left[\begin{array}{l}
X \\
Y \\
Z
\end{array}\right]=\left[\begin{array}{lll}
a_{1} & b_{1} & c_{1} \\
a_{2} & b_{2} & c_{2} \\
a_{3} & b_{3} & c_{3}
\end{array}\right]\left[\begin{array}{l}
X \\
Y \\
Z
\end{array}\right]=\frac{1}{\lambda}\left[\begin{array}{lll}
a_{1} & b_{1} & c_{1} \\
a_{2} & b_{2} & c_{2} \\
a_{3} & b_{3} & c_{3}
\end{array}\right]\left[\begin{array}{l}
X_{A}-X_{S} \\
-Y_{A}+Y_{S} \\
-Z_{A}+Z_{S}
\end{array}\right]
$$

We can relatively reorganize Equation 3 and obtain the collinear equation model fitting indoor environment as follows:

$$
\left\{\begin{array}{l}
x=-f \frac{a_{1}\left(\mathrm{X}_{A}-\mathrm{X}_{S}\right)+b_{1}\left(-\mathrm{Y}_{A}+Y_{S}\right)+c_{1}\left(-\mathrm{Z}_{A}+Z_{S}\right)}{a_{3}\left(\mathrm{X}_{A}-\mathrm{X}_{S}\right)+b_{3}\left(-\mathrm{Y}_{A}+Y_{S}\right)+c_{3}\left(-\mathrm{Z}_{A}+Z_{S}\right)} \\
y=-f \frac{a_{2}\left(\mathrm{X}_{A}-\mathrm{X}_{S}\right)+b_{2}\left(-\mathrm{Y}_{A}+Y_{S}\right)+c_{2}\left(-\mathrm{Z}_{A}+Z_{S}\right)}{a_{3}\left(\mathrm{X}_{A}-\mathrm{X}_{S}\right)+b_{3}\left(-\mathrm{Y}_{A}+Y_{S}\right)+c_{3}\left(-\mathrm{Z}_{A}+Z_{S}\right)}
\end{array}\right.
$$

The photo taken by the front camera is axisymmetric processed by imaging system for user's habits in positioning. As vision information has been considered, the model should be modified and the imaging relationship among object point, image point and photographic center should be changed while the collinear equation model in Equation 4 is the actually imaging system. The origin of photo coordinate system is in the center of image, so reverse the $\mathrm{x}$ axis for solving the case of axial symmetry. The modified collinear equation is shown as follows:

$$
\left\{\begin{array}{l}
x=f \frac{a_{1}\left(\mathrm{X}_{A}-\mathrm{X}_{S}\right)+b_{1}\left(-\mathrm{Y}_{A}+Y_{S}\right)+c_{1}\left(-\mathrm{Z}_{A}+Z_{S}\right)}{a_{3}\left(\mathrm{X}_{A}-\mathrm{X}_{S}\right)+b_{3}\left(-\mathrm{Y}_{A}+Y_{S}\right)+c_{3}\left(-\mathrm{Z}_{A}+Z_{S}\right)} \\
y=-f \frac{a_{2}\left(\mathrm{X}_{A}-\mathrm{X}_{S}\right)+b_{2}\left(-\mathrm{Y}_{A}+Y_{S}\right)+c_{2}\left(-\mathrm{Z}_{A}+Z_{S}\right)}{a_{3}\left(\mathrm{X}_{A}-\mathrm{X}_{S}\right)+b_{3}\left(-\mathrm{Y}_{A}+Y_{S}\right)+c_{3}\left(-\mathrm{Z}_{A}+Z_{S}\right)}
\end{array}\right.
$$

In Equation 5, $(x, y)$ is the image coordinate in two-dimensional image, $\left(\mathrm{X}_{A}, \mathrm{Y}_{A}, \mathrm{Z}_{A}\right)$ is the three-dimensional coordinate of LED light, and $\left(\mathrm{X}_{s}, \mathrm{Y}_{s}, \mathrm{Z}_{s}\right)$ is the coordinate of photographic center in the same coordinate system. $(\varphi, \omega, \kappa)$ are the rotation angles in three directions while imaging. $\left(a_{1}, a_{2}, a_{3}, b_{1}, b_{2}, b_{3}, c_{1}, c_{2}, c_{3}\right)$ is the rotation matrix which is expressed by three rotation angles. Equation 5 is the final form of modified mathematical model based on collinear equations.

As stated before, the photo imaging in the front camera with that imaging actually into the axial symmetry, so reverse the $\mathrm{x}$ axis while the center of photo coordinate system is in the center of image. Compared the mobile terminal with the LED coordinate system, it is distinct in ground three-dimensional coordinate system and photo auxiliary coordinate system that the $\mathrm{y}$-value and $\mathrm{z}$-value are opposite while $\mathrm{x}$ is being equal.

\section{SIMULATION AND ANALYSIS}

\subsection{Simulation experiments}

A hardware-in-loop simulation is used for the test of collinear equation in this paper. First, parameters of testing ground including position of terminals are calibrated for precision analysis subsequently. Three-dimensional coordinates of LED lights are obtained through the calibration of LED lights. Then image information of LED lights is collected by the mobile terminal front camera. After matching the three-dimensional coordinates of LED lights with the image information acquired from image extraction and recognition of LED lights, we compute the coordinate of terminal. Finally, the test result including the overall accuracy of positioning should be analyzed. Also, the influence of distribution and numbers of LED lights on accuracy of positioning is discussed.

(1) Parameters calibration of testing ground

The location of LED lights and the terminal should be calibrated for testing the validity of collinear equation model. First, the location of terminal that will collect the vision information should be confirmed by establishing the relative coordinate system. Then the distance between the terminal point and the LED lights is obtained by a laser DME (Distance Measurement Equipment). Compute the location information with space intersection.

The least squares estimation is used through introducing the aforementioned point for diminishing error of calibration caused in measurement. Regard the three-dimensional coordinates of LED lights as the 
truth-value in the relative coordinate system.

The scene of simulation is shown as follows. The distance between LED lights is $0.6 \mathrm{~m}$ in $\mathrm{x}$ axis, and $0.6 \mathrm{~m}$ in y axis. The height of building is $2.8 \mathrm{~m}$. Terminals are used to take photos in the points of $0.8 * 0.8 \mathrm{~m}$ grid.

(2) Visual information collection

Two phone terminals with different focuses are used for the image-collecting through their front cameras. We acquire four sets of data by using different terminals on different heights to test the universality of model. We use the terminals that focuses are respectively $2 \mathrm{~mm}$ and $3 \mathrm{~mm}$ to take photos at the height of $1 \mathrm{~m}$ and $2.143 \mathrm{~m}$. By using Matlab, the coordinate of terminal is acquired with collinear equation model, and the accuracy of positioning is analyzed.

\subsection{Result analysis of simulation}

After calibrating the scene and the data acquisition, we compare the result and the position of the actual terminal, and testify the collinear equation model taken by the mobile front camera with the algorithm model mentioned in the Equation 5. We obtain 172 terminal positions through four groups of actual experimental data, and then we calculate the deviation of the ground to the calibrated spot on the ground. This chapter is firstly analyzed from the whole page to locate the accuracy, and then we analyze the effect of the positioning accuracy from the following 2 aspects, which are the distribution of LED lights in the picture, and the amounts of LED lights involved in the calculation.

(1) Distribution of accuracy and statistics of the whole positioning

Comprehensively evaluate the accuracy of the positioning of the scene, simultaneously, calculate the 172 positioning errors of different sight scenes and terminals. All the LED lights captured by each spot are members of the calculation. As the differentiation of the angle of the sight scene, the amounts of the LED lights in the image, which are four at least or 20 at most, are not the same.

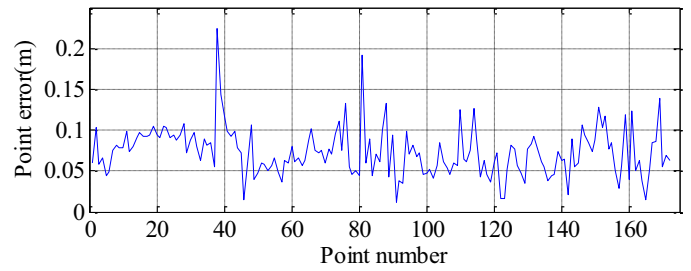

Figure 3. Position deviation of the positioning result

According to Figure 3, the deviation's range is mostly from $0.05 \mathrm{~m}$ to $0.1 \mathrm{~m}$, and its average is $0.073 \mathrm{~m}$, the minimum is $0.012 \mathrm{~m}$, and the maximum is $0.223 \mathrm{~m}$. In addition, to judge the regular pattern of the distribution of the errors as well as to count the accumulative distribution of the errors, we have drawn the CDF (Cumulative Distribution Function) as shown in Figure 4. The horizontal axis represents the number of the positioning errors, and the vertical axis shows the probability which is less than or equal to the very number corresponding to horizontal axis. It turns out that the slope of the curve remains high between the positioning errors which are from $0.04 \mathrm{~m}$ to $0.1 \mathrm{~m}$, that is, there are plenty of the terminals accounting results in the deviation interval, and the percentage which is less than $0.093 \mathrm{~m}$ reaches to $80 \%$. Yet the percentage of deviation which is more than $0.1 \mathrm{~m}$ is small, the curve tends to be steady after turning to $0.12 \mathrm{~m}$.

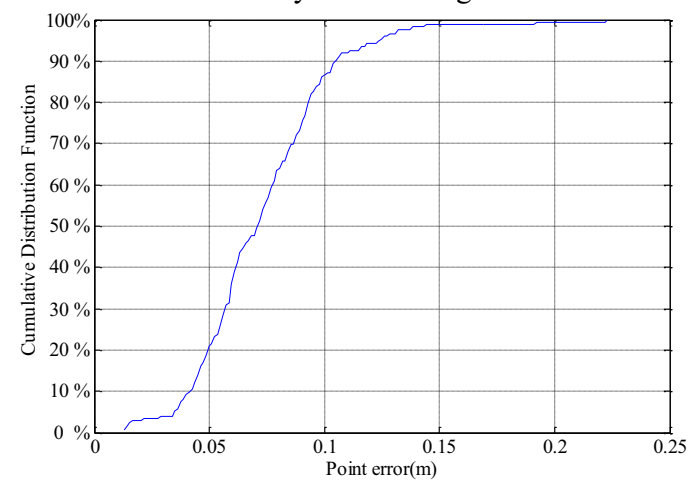

Figure 4. Cumulative distribution function of errors

The positioning accuracy of the whole page has shown the availability of the improvement in the collinear equation model. The positioning accuracy could be around $7 \mathrm{~cm}$ with different termination, complicated environment, and mutual restraint.

(2) Effects of distribution of LED lights on the positioning accuracy

To obtain a better analysis on the effect of the distribution of LED lights to accuracy, further testify the positioning results of the testing algorithm and lay foundations for the follow-up analysis of deviation, we pick the sight information of focal length which is $2 \mathrm{~mm}$, height which is $1 \mathrm{~m}$ and the responding three-dimensional axis data of LED to do it. Each image of this group maintains large information, including at least the three-dimensional axis data and two-dimensional image data of 9 LED lights, and we choose the data of 6 LED lights of each image to analysis. However, the distribution patterns are different. One is randomly picked from 6 lights in the image, while the other is evenly distributed. The results are shown as follows.

The distribution deviation between the random one and the even-distributed ones ranges from $0.05 \mathrm{~m}$ to $0.15 \mathrm{~m}$. Therefore, we draw a CDF which is shown in Figure 5. The positioning errors of LED lights which are even-distributed is far better than the random one, and $80 \%$ of the positioning errors are less than $0.128 \mathrm{~m}$, yet the random ones are $0.139 \mathrm{~m}$.

As mentioned above, the positioning accuracy cor- 
responds to the positioning of brought data in the image. When the LED lights positioning responds to the data which is evenly distributed in the image, the accuracy is better than the random one. In a result, we should make reasonable arrangements to the LED lights to obtain the best structure of calculation.

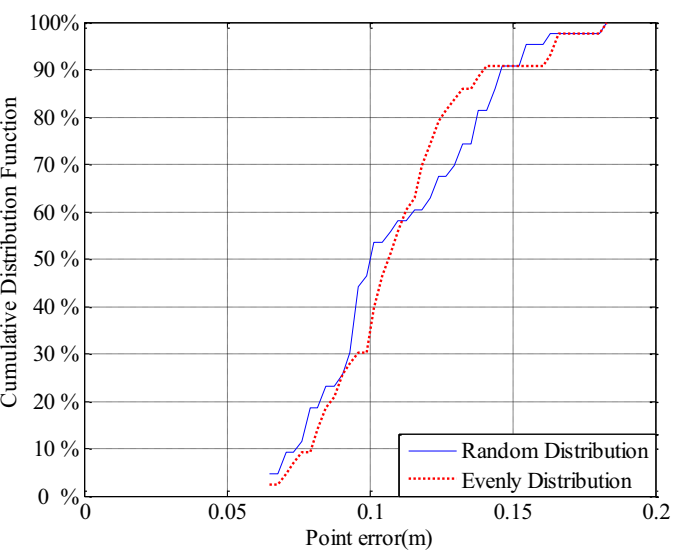

Figure 5. Cumulative distribution function of deviation

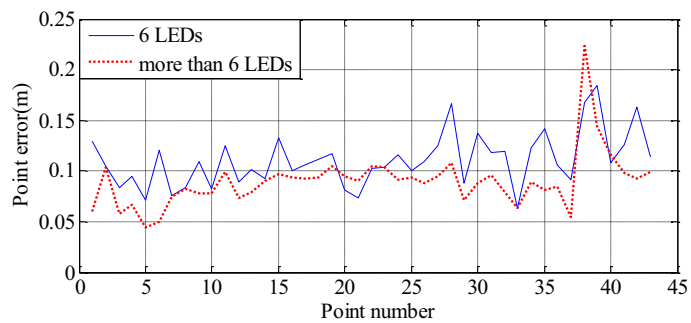

Figure 6. Errors of positioning results of different amounts of LED lights

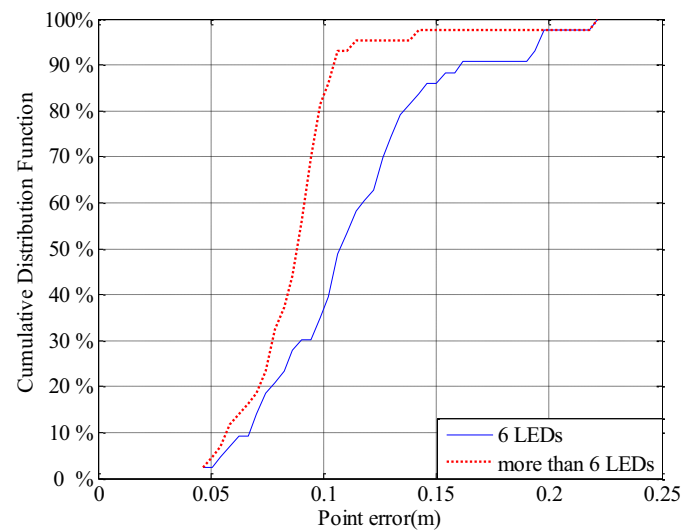

Figure 7. Cumulative distribution function of deviation
(3) Effects of the amount of LED lights on accuracy We pick the visual information of focal length which is $2 \mathrm{~mm}$, height which is $1 \mathrm{~m}$ and the responding three-dimensional position of LED to analyze. Each of the images is evenly distributed with different amount One is evenly with 6 lights, and the other is evenly more than 6 lights. The results are shown as follows:

As shown in Figure 6, the positioning error with different amount is in a range from $0.05 \mathrm{~m}$ to $0.15 \mathrm{~m}$.

We can learn from the analysis mentioned earlier that the positioning accuracy corresponds to the position of data in the image and amounts. Generally, the better the quality of data is, the more the amounts will be and the higher the accuracy will be. However, the positioning accuracy will be affected when cross error appears.

\section{CONCLUSION}

A kind of collinear equation model of visual positioning using visible light communication is brought out in this essay. The input information uses two sorts of sensors of different resolution to extract and combine the visible light communication and visual information. A hardware-in-loop simulation has been conducted to verify the collinear equation. The three-dimensional positioning error is at the level of decimeter. For the LED lights, the distribution and the structure of groups affect the acquisition of visual information as well as the mutual restraint from the sight scene angle. The method could be transferred to cell phone application programs. When conducting physical experiments with external PD array, the availability in actual circumstances could be further tested.

\section{ACKNOWLEDGEMENT}

This paper is sponsored by The National Natural Science Foundation of China (No. 91438207).

\section{REFERENCES}

[1] Nakajima M. \& Haruyama S. 2013. New indoor navigation system for visually impaired people using visible light communication. EURASIP Journal on Wireless Communications and Networking, (1): 1-10.

[2] Jung S Y, Hann S. \& Park C S. 2011. TDOA-based optical wireless indoor localization using LED ceiling lamps. Consumer Electronics, IEEE Transactions on, 57(4): 1592-1597.

[3] Yang S H, Jeong E M. \& Kim D R, et al.2013. Indoor three-dimensional location estimation based on LED visible light communication. Electronics Letters, 49(1): 54-56.

[4] Hann S, Kim J H. \& Jung S Y, et al. 2010. White LED ceiling lights positioning systems for optical wireless 
indoor applications[C]//Optical Communication (ECOC), 2010 36th European Conference and Exhibition on. IEEE: 1-3.

[5] Kim B Y, Cho J S. \& Park Y, et al. 2012. Implementation of indoor positioning using LED and dual PC cameras[C]//Ubiquitous and Future Networks (ICUFN), 2012 Fourth International Conference on. IEEE: 476-477.

[6] Lee S J, Yoo J H. \& Jung S Y. 2012. VLC-based indoor location awareness using LED light and image sensors[C]//Photonics Asia. International Society for Optics and Photonics: $85580 \mathrm{~L}-85580 \mathrm{~L}-6$. 